\title{
Theoretical description of pressure-induced phase transitions: a case study of Ti-V alloys
}

Igor Abrikosov, A. V. Ponomareva, A. Yu. Nikonov, A. M. Zharmukhambetova, Igor Mosyagin, A. V. Lugovskoy, Olle Hellman, Hans Lind, A. I. Dmitriev and S. A. Barannikova

\section{Linköping University Post Print}

\section{Tweet}

N.B.: When citing this work, cite the original article.

This is an electronic version of an article published in:

Igor Abrikosov, A. V. Ponomareva, A. Yu. Nikonov, A. M. Zharmukhambetova, Igor Mosyagin, A. V. Lugovskoy, Olle Hellman, Hans Lind, A. I. Dmitriev and S. A. Barannikova, Theoretical description of pressure-induced phase transitions: a case study of Ti-V alloys, 2015, High Pressure Research, (35), 1, 42-48.

High Pressure Research is available online at informaworldTM:

http://dx.doi.org/10.1080/08957959.2014.992896

Copyright: Taylor \& Francis: STM, Behavioural Science and Public Health Titles http://www.tandf.co.uk/journals/default.asp

Postprint available at: Linköping University Electronic Press http://urn.kb.se/resolve?urn=urn:nbn:se:liu:diva-114586 


\title{
Theoretical description of pressure induced phase transitions: a case study of
}

\section{Ti-V alloys}

\author{
I. A. Abrikosov ${ }^{1,2}$, A. V. Ponomareva ${ }^{1}$, A. Yu. Nikonov ${ }^{3,4}$, A. M. Zharmukhambetova ${ }^{3}$, \\ I. Yu. Mosyagin ${ }^{1,2}$, A. V. Lugovskoy ${ }^{1}$, O. Hellman², H. Lind ${ }^{2}$, A. I. Dmitriev ${ }^{3,4}$, S. A. \\ Barannikova $^{3,4}$
}

\author{
${ }^{1}$ Materials Modeling and Development Laboratory, NUST "MISIS”, Moscow, Russia \\ ${ }^{2}$ IFM, Linköpings University, Linköping, Sweden \\ ${ }^{3}$ National Research Tomsk State University, Tomsk, Russia. \\ ${ }^{4}$ Institute of Strength Physics and Materials Science, SB RAS, Tomsk, Russia.
}

\begin{abstract}
We discuss theoretical description of pressure induced phase transitions by means of first-principles calculations in the framework of Density Functional Theory (DFT). We illustrate applications of theoretical tools that allow one to take into account configurational and vibrational disorder, considering Ti-V alloys as a model system. The universality of the first-principles theory allows us to apply it in studies of remarkably reach physics in Ti-V system upon compression. Besides the transitions between different crystal structures, we discuss isostructural transitions in bcc Ti-V alloys. Moreover, we present arguments for possible electronic transitions in this system, which may explain peculiar behaviour of elastic properties of $\mathrm{V}$ upon compression.
\end{abstract}

Keywords: first-principles calculations; phase stability; high pressure; Ti-V alloys

\section{Introduction}

Materials properties and their performance in applications are determined by chemical composition, temperature, and pressure. Their variation can give rise to phase transitions, a fascinating problem for fundamental science and an important tool for the materials design. Therefore there is very high interest in developing qualitative understanding, as well as in obtaining quantitative theoretical description of pressure induced phase transitions. The phenomenological theory of phase transitions follows from Gibbs equilibrium thermodynamics. At fixed pressure $P$ and temperature $T$ a system is described by the Gibbs free energy $G=E+P V-T S$, where $E$ is the potential energy of the system, $S$ denotes the entropy, and $V$ represents volume. On the other hand, for the description of the phase stability by means of the modern first-principles theory of alloys one most often uses the Helmholtz free energy $F=E-T S$ [1]. Thus, the $P V$ term is often left off the theory. A justification for this approach comes from the fact that for equilibria involving only solid and liquid phases, the phase boundaries are typically shifted only by the order of a few hundredths of a degree per bar change in $P$, and therefore the effect of $P V$-term upon the phase transitions temperature is often assumed to be negligible [2]. At the same time, for instance, stress or pressure levels in modern cutting operations can be as high as several GPa at the cutting edge of the tools [3]. In the Earth's core studies one deals with pressures up to $360 \mathrm{GPa}$ and temperatures somewhere from $4000 \mathrm{~K}$ to $7000 \mathrm{~K}$ [4].

Obviously, the importance of $P V$-term is well recognized in high-pressure physics. However, here the studies are often focused on the properties of monoatomic solids or ordered compounds. If phase $\alpha$ transforms into phase $\beta$ in a pure material at equilibrium transformation temperature $T_{c}, G_{\alpha}=G_{\beta}$ at $T_{c}$. But for complex multicomponent systems, the chemical potential $\mu_{I}$ must be included, and at the equilibrium the Gibbs-Duhem relation holds:

$$
S d T-V d P+\sum_{I} n_{I} \mu_{I}=0
$$


An important consequence here is that going from pure elements to alloys in studies of pressure induced phase transitions requires a modification of the approach, in which free energies of systems with different crystal structures are directly compared to each other to a proper consideration of alloy thermodynamics [1], which allows for a coexistence of phases with different compositions.

In this work we illustrate applications of theoretical tools that allow one to take into account temperature induced configurational and vibrational disorder in studies of pressure induced phase transitions of Ti-V alloys. We limit ourselves by three close-packed phases, fcc, hcp, and bcc. In particular, we do not consider $\omega$ phase. Ti-based alloys are chosen as a model system because they are important technological materials, e.g. for aerospace and medical applications. Moroover, they exhibit several types of temperature and pressure induced phase transitions, which make them attractive objects for fundamental studies.

\section{Influence of pressure on the lattice stability of Ti-V alloys}

Let us start with lattice stability of Ti-V alloys, estimated at zero temperature. The concept of lattice stability introduced by L. Kaufman [5] refers to the differences in the Gibbs energies between different crystal structures of pure elements. The data base of lattice stabilities at $\mathrm{T}=0 \mathrm{~K}$, given by the structural enthalpy differences between different crystal structures $\Delta H$ (where $H=E+P V$ ) is the corner stone of a powerful tool for calculations of phase diagrams, the so-called CALPHAD approach. The concept is easily generalized for the case of alloys, and it provides the understanding of phase transformations at fixed composition, like martensitic phase transformations or phase transitions in experiments, where the diffusion is artificially suppressed.

DFT calculations of the enthalpies for bcc, fcc, and hcp Ti-V alloys were carried out using the exact muffin-tin orbital (EMTO) method combined with the coherent potential approximation (CPA) [6]. The methodology is known as an efficient tool for theoretical simulations of random alloys, though the absolute values should be taken with some care because of the neglect of the contribution from the local distortions of the crystal lattice in the CPA. Also, recently it has been demonstrated that potential energies in systems with mechanical or dynamic instabilities may depend strongly on the temperature [7]. As will be shown in Secs. 4 and 5, these are important issues for Ti-V system. However, neither of these effects should influence the calculated trends, which are of primary importance for this study, and which are reliably predicted by the EMTO-CPA calculations, as will be shown below.
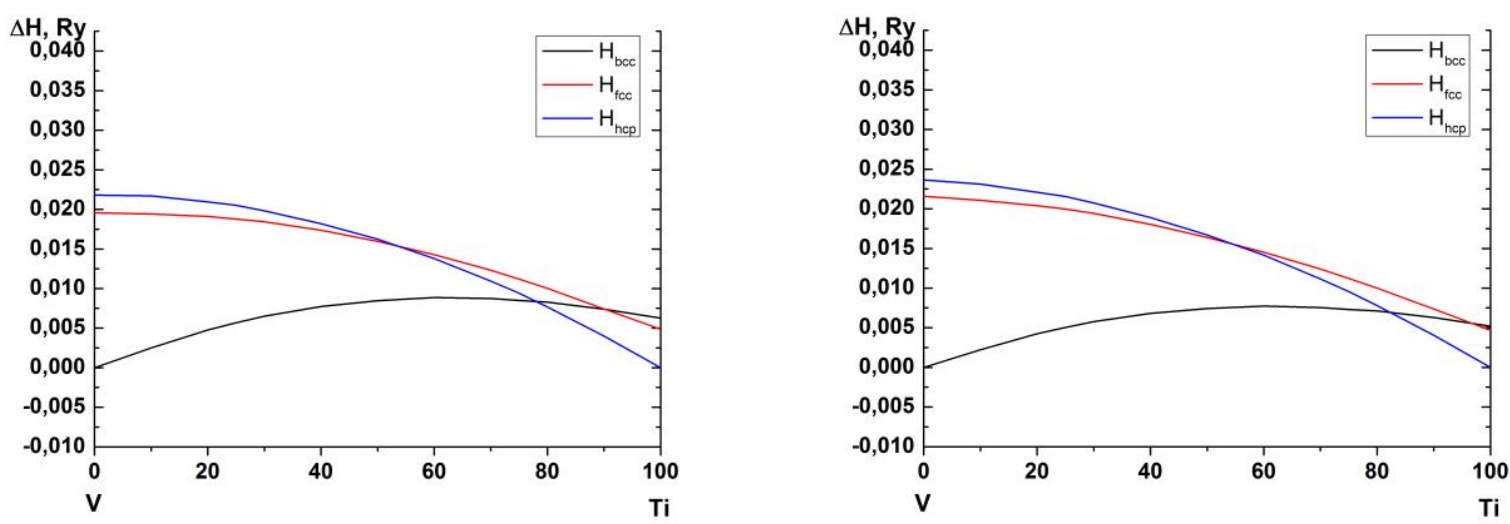

Figure 1 (colour online). Calculated total mixing enthalpies $\Delta H_{V_{1-x} T i_{x}}^{\text {mix,tol }}$ for bcc (black lines), fcc (red lines) and hcp (blue lines) Ti-V alloys at $\mathrm{P}=0 \mathrm{GPa}$ (left panel) and $\mathrm{P}=10 \mathrm{GPa}$ (right panel) as a function of Ti concentration (in at. \%). 
The accuracy of the calculated lattice stabilities for the pure elements Ti and V using the EMTO method in comparison to other first-principles methods has been demonstrated in [8]. To facilitate the understanding of the stability trends in Ti-V alloys, we express the results in the form of total mixing enthalpies:

$$
\Delta H_{A_{1-x} B_{x}}^{\text {mix,tal }}=H_{A_{1-x} B_{x}}-(1-x) H_{A}-x H_{B}
$$

where $H_{A(B)}$ are the enthalpies of the pure alloy components, calculated in their respective ground state crystal structures. The results are shown in Fig. 1. Unfortunately, we are unable to find any experimental information on the mixing enthalpies for this system, even at ambient pressure. As a matter of fact, this underlines the importance of theoretical simulations.

At the same time, our calculations reproduce known experimental trends in Ti-V system. Indeed, fcc Ti-V alloys always have higher enthalpies, at least within the pressure range considered in this work. This is in agreement with experiment. We therefore will not discuss them in the remaining part of this paper. At low temperature Ti-V system is characterized by a tendency towards a phase separation into hcp Ti-rich and bcc V-rich phases, in complete agreement with the behaviour of the mixing enthalpy shown in Fig. 1. As a matter of fact, this underlines the necessity to considering phase equilibria in alloys, allowing for coexistence of phases with different compositions. But in terms of the lattice stability problem, the enthalpy difference between bcc and hcp Ti-V alloys decreases rapidly when $\mathrm{V}$ is added to $\mathrm{Ti}$ (Fig. 1). This is in agreement with experiment as well. Indeed, $\mathrm{V}$ is known as a $\beta$-Ti stabilizer (it stabilizes the bcc phase).

Importantly, this tendency becomes stronger with increasing pressure. Considering phase transitions at fixed alloy composition, the bcc phase is getting more favourable with respect to the hcp phase from the enthalpy point of view at about 22 at. \% of $\mathrm{V}$ at ambient pressure. At $10 \mathrm{GPa}$ the crossover occurs at smaller concentration $\sim 18$ at. \% V (Fig. 1). The observed stabilization of the bcc phase can be explained by the electronic structure effects in the framework of the so-called canonical $d$-band model [9]. According to it Ti, being within the hcp-phase stability field, is close to the hcp-bcc boundary. Thus, increasing number of $d$-electrons in the system should stabilize the bcc phase. Because $\mathrm{V}$ has more electrons, alloying it with $\mathrm{Ti}$ increases the $d$-band filling. Moreover, pressure increases it even further due to the $s$ - to $d$-electrons charge transfer mechanism. Thus, both, the alloying of $\mathrm{Ti}$ with $\mathrm{V}$ and the application of pressure stabilize the bcc phases in Ti-V system.
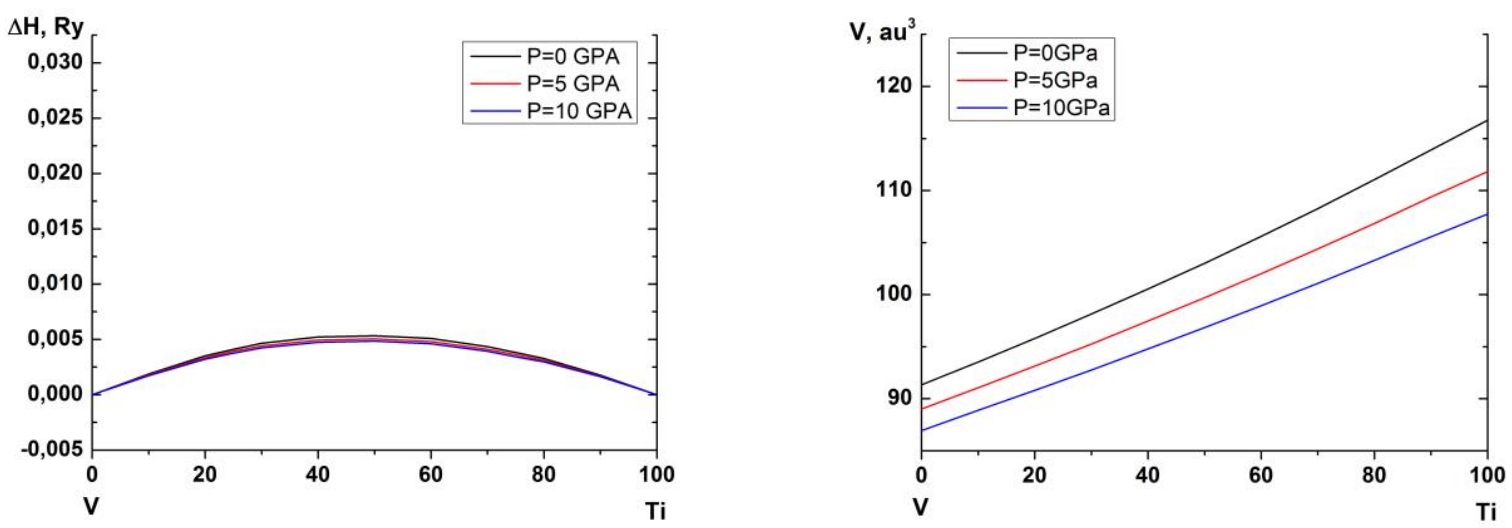

Figure 2 (colour online). Calculated isostructural mixing enthalpies $\Delta H_{V_{1-x} T_{i_{x}}}^{\text {mix,iso }}$ (in Ry/atom, left panel) and volume per atom $V$ (in atomic units, right panel) for bcc Ti-V alloys at $\mathrm{P}=0$ (black lines), $5 \mathrm{GPa}$ (red lines) and $10 \mathrm{GPa}$ (blue lines) as a function of Ti concentration (in at. \%). 


\section{Influence of pressure on the stability of solid solution phase against isostructural decomposition}

Isostructural phase transitions are common in systems with a tendency towards phase separation. They can proceed via spinodal, as well as via nucleation and growth mechanisms. In Ti-V systems bcc solid solutions are of interest in this respect. The experimental situation here is somewhat controversial: one can find phase diagrams with or without the isostructural phase separation line. In Fig. 2 we show the isostructural mixing enthalpy $\Delta H_{V_{1-x} T i_{x}}^{m i x}$ of bcc Ti-V alloys. It is defined by the equation similar to Eq. (2), but with $H_{A(B)}$ calculated in the same crystal structures, bcc in our case.

One can see that $\Delta H_{V_{1-x} T_{i}}^{m i x, i s o}$ is positive, indicating a tendency towards the phase separation. The strength of the clustering could be somewhat overestimated due to the neglect of the local lattice relaxations effect in the CPA, which should make the enthalpy less positive. On the other hand, the clustering effects are believed to be present in bcc Ti-V alloys, in agreement with our calculations. But relatively small values of $\Delta H_{V_{1-x} T_{i}}^{\text {mix } i s}$ may explain the above mentioned controversy between different Ti-V phase diagrams. Of higher interest for this study is to consider the effect of pressure on the stability of solid solution phases against the isostructural decomposition. As a matter of fact, using the model proposed by Alling et al. in [10], one can understand it from deviations of the concentration dependence of atomic volumes from the linear behaviour (from the Zen's law) [11]. Alling et al. showed that negative (positive) deviations of $V$ from the Zen's law should lead to more negative (positive) values of the mixing enthalpy, thus making the alloy more (less) stable. In the right panel of Fig. 3 one sees that $V$ in Ti-V system follows the Zen's law very well. Thus, the effect of pressure on $\Delta H_{V_{1-x} T_{x}}^{m i x, i s}$ is expected to be quite weak. This is essentially what is seen in our firstprinciples calculations, left panel in Fig. 2.

\section{Influence of pressure on the mechanical stability}

Nowadays it is well recognized that the analysis of thermodynamic stability of alloys, considered in Secs. 2 and 3 should be complemented by an analysis of their mechanical and dynamical stability. Below we carry it out for the end member elements, pure V and Ti. Let us start with the former. For cubic crystals the mechanical stability criterion is given by the following relation between its elastic moduli:
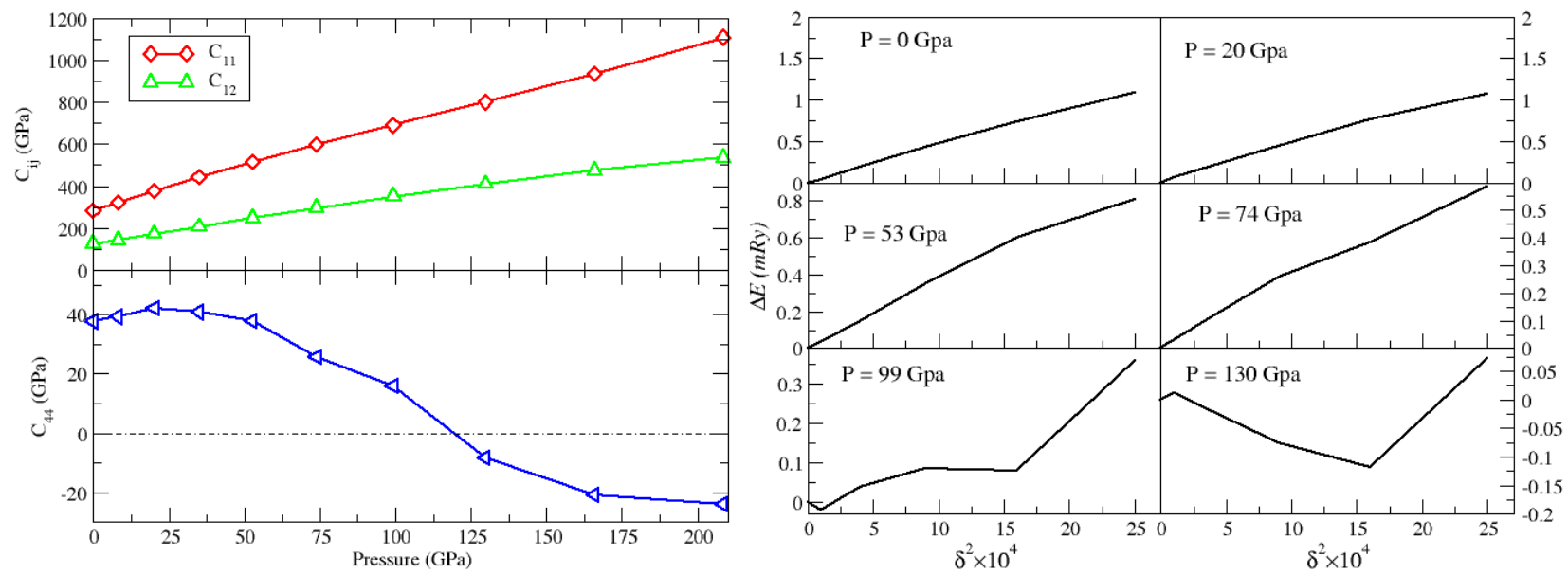

Figure 3 (colour online). (left panel) Calculated pressure dependence of elastic moduli (in GPa) $C_{11}$, $C_{12}$, and $C_{44}$ for bcc $\mathrm{V}$ as a function of pressure. (Right panel) Variation of total energy $E$ of bcc $\mathrm{V}$ as a function of square of monoclinic distortion $\delta^{2}$, used for calculations of $C_{44}$ at different pressure. 
$C_{44}>0, \quad C_{11}>\left|C_{12}\right|, \quad C_{11}+2 C_{12}>0$.

In this work the pressure dependence of the moduli were calculated by the EMTO method as described in [8]. The results are shown in Fig. 3. The left panel confirms that our calculations are in good agreement with earlier calculations [12]. BCC V is mechanically stable at ambient pressure, but it becomes unstable according to Eq. (3) around $125 \mathrm{GPa}$ in our calculations. The transition is well described in the literature [12]. Going beyond the known facts, we analyse it in more details. In the right panel of Fig. 3 we show variation of the total energy $E$ of bcc $\mathrm{V}$ as a function of square of distortion, $\delta^{2}$, used for calculations of the elastic moduli [12] (in case of $C_{44}$ a monoclinic distortion is used). Usually, the dependence is linear for $\delta$ within the elastic limit. This is indeed the case for bcc $\mathrm{V}$ at low pressure, where it is mechanically stable. However, the behaviour becomes really peculiar when the transition pressure is approached. In principle, singularities of elastic constants may indicate the presence of so-called electronic topological transition, associated with a change of the Fermi surface topology [13]. Clearly, the peculiar behaviour $E$ vs $\delta^{2}$ seen in Fig. 3 deserves further, more detailed study.

\section{Theoretical description of temperature induced dynamical stabilization of bcc Ti}

As a matter of fact, mechanical instability considered in Sec. 4 represents just a particular case of a more general phenomenon, the dynamical instability. The latter manifests itself in calculations by the appearance of imaginary frequencies on the phonon spectra of unstable materials, like shown in the left panel of Fig. 4 for bcc Ti. The imaginary frequencies indicate that any perturbation with the corresponding wave vector should destroy the bcc structure of $\mathrm{Ti}$, and therefore it cannot exist. However, these results are obtained at $\mathrm{T}=0 \mathrm{~K}$, while bcc $\mathrm{Ti}$ is stable only at high temperature, and it is well accepted that it is stabilized by anharmonic effects. To deal with such strongly anharmonic system as bcc Ti, Helmann et al. have developed a novel approach, the temperature dependent effective potential method (TDEP) [15,16]. We have applied TDEP to simulate the phonon dispersion relations of bcc $\mathrm{Ti}$ at $\mathrm{T}=1500 \mathrm{~K}$. The methodological details have been the same as in $[15,16]$. The results are shown in the right panel of Fig. 4, together with experimental spectrum from [14]. One can clearly see that TDEP is capable to capture the transition from dynamical instability to dynamical stability in bcc Ti upon its heating. Moreover, the calculated phonon dispersion relations are in very good agreement with experiment.
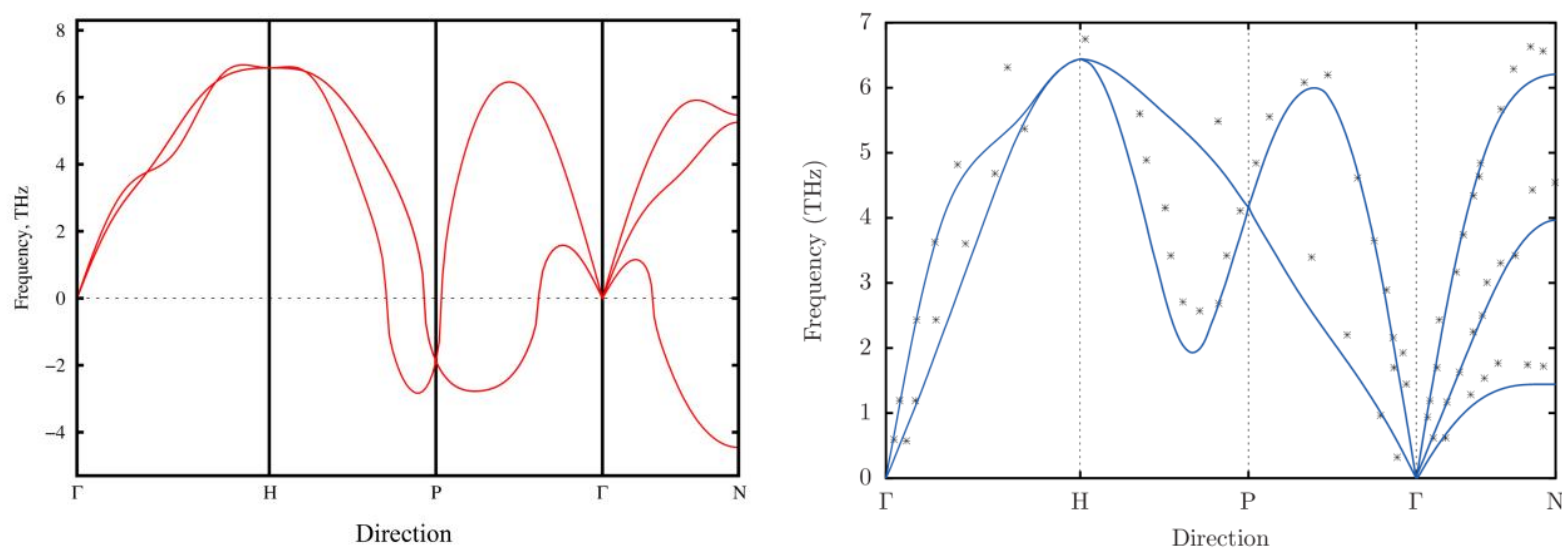

Figure 4 (colour online). Phonon dispersion relations for bcc Ti, calculated within the state-of-theart quasiharmonic approximation at $\mathrm{T}=0 \mathrm{~K}$ (left) and with temperature dependent effective potential method at $\mathrm{T}=1500 \mathrm{~K}$ (right). Negative frequencies denote imaginary numbers. Experimental results from Ref. [14] are shown in the right panel by stars. They are obtained at $\mathrm{T}=1293 \mathrm{~K}$. 


\section{Summary}

By means of first-principles calculations we have investigated the influence of pressure on structure and stability of Ti-V system, considering effects of configurational and vibrational disorder. We have observed that the application of the pressure has stabilized the bcc phases of Ti-V alloys relative to the hcp phase, but has little effect on the isostructural decomposition of bcc solid solutions. We have explained the former by the filling of Ti $d$-band upon the compression, and the latter by using the thermodynamic model of Alling et al. [10]. Considering the pressure dependence of elastic moduli of bcc V, we have presented arguments for possible electronic transitions in this system. Considering temperature induced stabilization of bcc $\mathrm{Ti}$, we have illustrated applicability of a novel method, TDEP, for simulations of strongly anharmonic solids. Its implementation for the case of solid solution phases will greatly strengthen capability of theory to study pressure induced phase transitions in complex materials.

\section{Acknowledgements}

This work was supported by the Grant of Russian Federation Ministry for Science and Education (grant no. 14.Y26.31.0005). SSF grant SRL 10-0026, Russian Foundation for Basic Researches grant No. 13-02-00606a and the Program of Fundamental Research of State Academies of Sciences for the period 2013-2020 years are gratefully acknowledged.

\section{References}

[1] Ruban AV and Abrikosov IA. Configurational thermodynamics of alloys from firstprinciples: Effective cluster interactions. Rep. Prog. Phys. 2008; 71: 046501.

[2] Pelton AD, Phase Diagrams. Physical Metallurgy, Amsterdam: North-Holland, 1996; 471-533.

[3] M'Saoubi R, Ruppi S. Wear and thermal behavior of CVD alpha-Al203 and MTCVD Ti(C,N) coatings during machining. CIRP Ann. 2009; 58:57.

[4] Dubrovinsky L, Dubrovinskaia N, Narygina O, Kuznetzov A, Prakapenka V, Vitos L, Johansson B, Mikhaylushkin AS, Simak SI and Abrikosov IA. Body-Centred-Cubic Iron-Nickel Alloy in Earth's Core. Science 2007; 316:1880.

[5] Kaufman L and Bernstein H. Computer Calculations of Phase Diagrams. Academic, New York, 1970.

[6] Vitos L, Abrikosov I A and Johansson B. Anisotropic lattice distortions in random alloys from first-principles theory 2001; Phys. Rev. Lett. 87:156401.

[7] Asker C, Belonoshko AB, Mikhaylushkin AS and Abrikosov IA. First-principles solution to the problem of Mo lattice stability. Phys. Rev. B 2008; 77: 220102.

[8] Abrikosov I A, Nikonov A Yu, Ponomareva A V, Dmitriev A I and Barannikova S A. Theoretical Modeling of Thermodynamic and Mechanical Properties of the Pure Components of Ti and Zr Based Alloys Using the Exact Muffin-Tin Orbitals Method. Russian Physics Journal 2014; 56(9):1030.

[9] Skriver HL. Crystal-structure from one-electron theory. Phys. Rev. B. 1985, 31:1909.

[10] Alling B, Oden M, Hultman L and Abrikosov I A. Pressure enhancement of the isostructural cubic decomposition in Ti1-xAlxN. Appl. Phys. Lett. 2009; 95: 181906.

[11] Zen E-an. Validity of Vegard's Law. Am. Mineral. 1956; 41: 523.

[12] Hammerschmidt T, Abrikosov I A, Alfe D, Fries SG, Höglund L, Jacobs M H G, Koßmann J, Lu X-G, Paul G. Including the effects of pressure and stress in thermodynamic functions. Phys. Stat. Sol. (b) 2014; 251: 81 , and references wherein.

[13] Lifshitz IM. Anomalies of electron characteristics of a metal in the high pressure region. Sov. Phys. JETP 1960;

11: 1130 .

[14] Petry W, Heiming A, Trampenau J, Alba M, Herzig C, Schober HR, and Vogl G. Phonon-dispersion of the bcc phase of group-IV metals. I. bcc titanium. Phys. Rev. B 1991; 43:10933.

[15] Hellman O, Abrikosov IA and Simak SI. Lattice dynamics of anharmonic solids from first principles. Phys. Rev. B 2011; 84:180301(R);

[16] Hellman O, Steneteg P, Abrikosov IA, and Simak SI. Temperature dependent effective potential method for accurate free energy calculations of solids. Phys. Rev. B. 2013; 87: 104111. 